\title{
Implementation of the ATLAS trigger within the ATLAS Multi-Threaded Software Framework: AthenaMT
}

\section{IEEE eScience}

29 October - 1 November 2018, Amsterdam

Tim Martin, University of Warwick

On behalf of the ATLAS Collaboration

Tim. Martin@cern.ch

Slides: http: //cern.ch/go/97FW

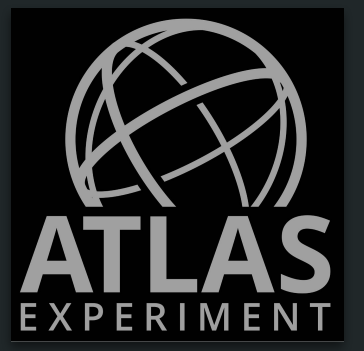




\section{ATLAS Trigger \& Data Acquisition}

Event rates

(peak)

Trigger

DAQ

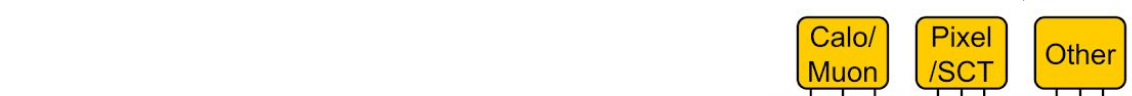

Hardware Dominant Domain

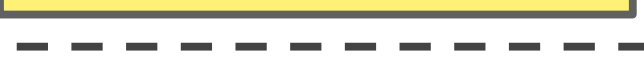

Software Dominant Domain

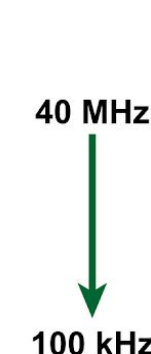

$100 \mathrm{kHz}$ 1
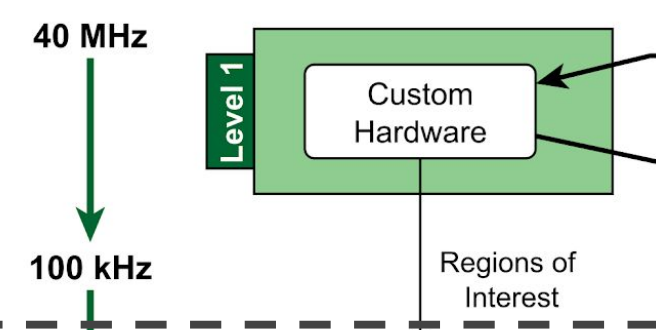

Interest

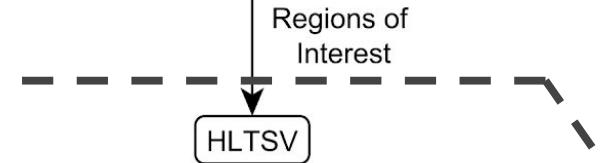

Focus of today's presentation
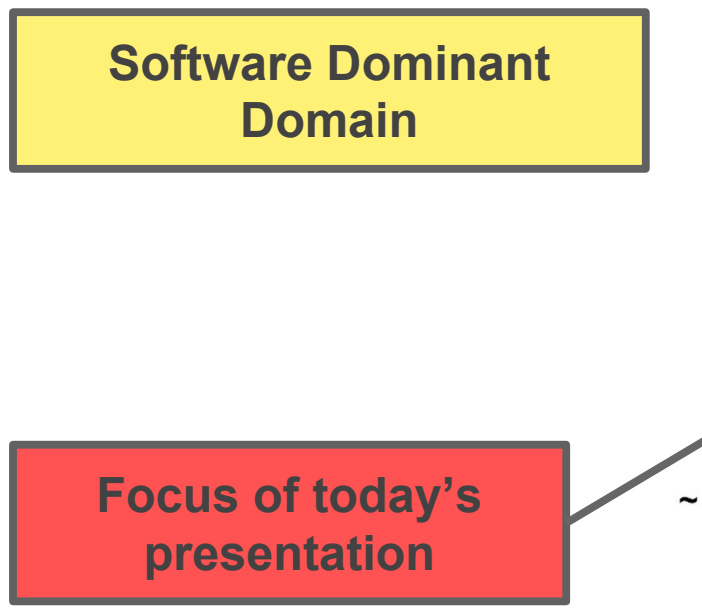

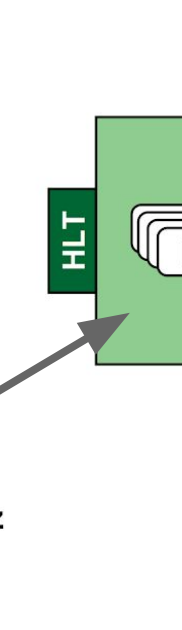

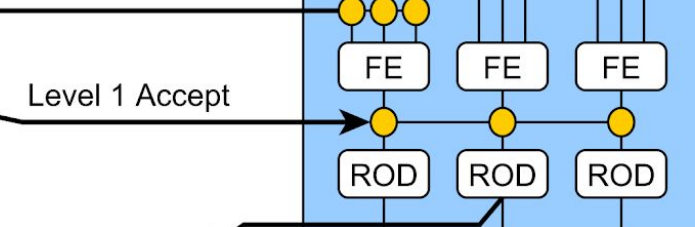

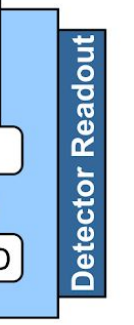

Peak data rates (primary physics)

$O(60 \mathrm{~TB} / \mathrm{s})$

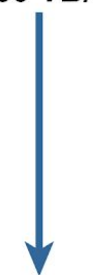

$\sim 160 \mathrm{~GB} / \mathrm{s}$

$\sim 25 \mathrm{~GB} / \mathrm{s}$

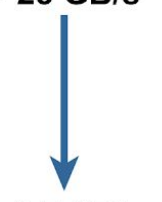

$\sim 1.5 \mathrm{~GB} / \mathrm{s}$ 


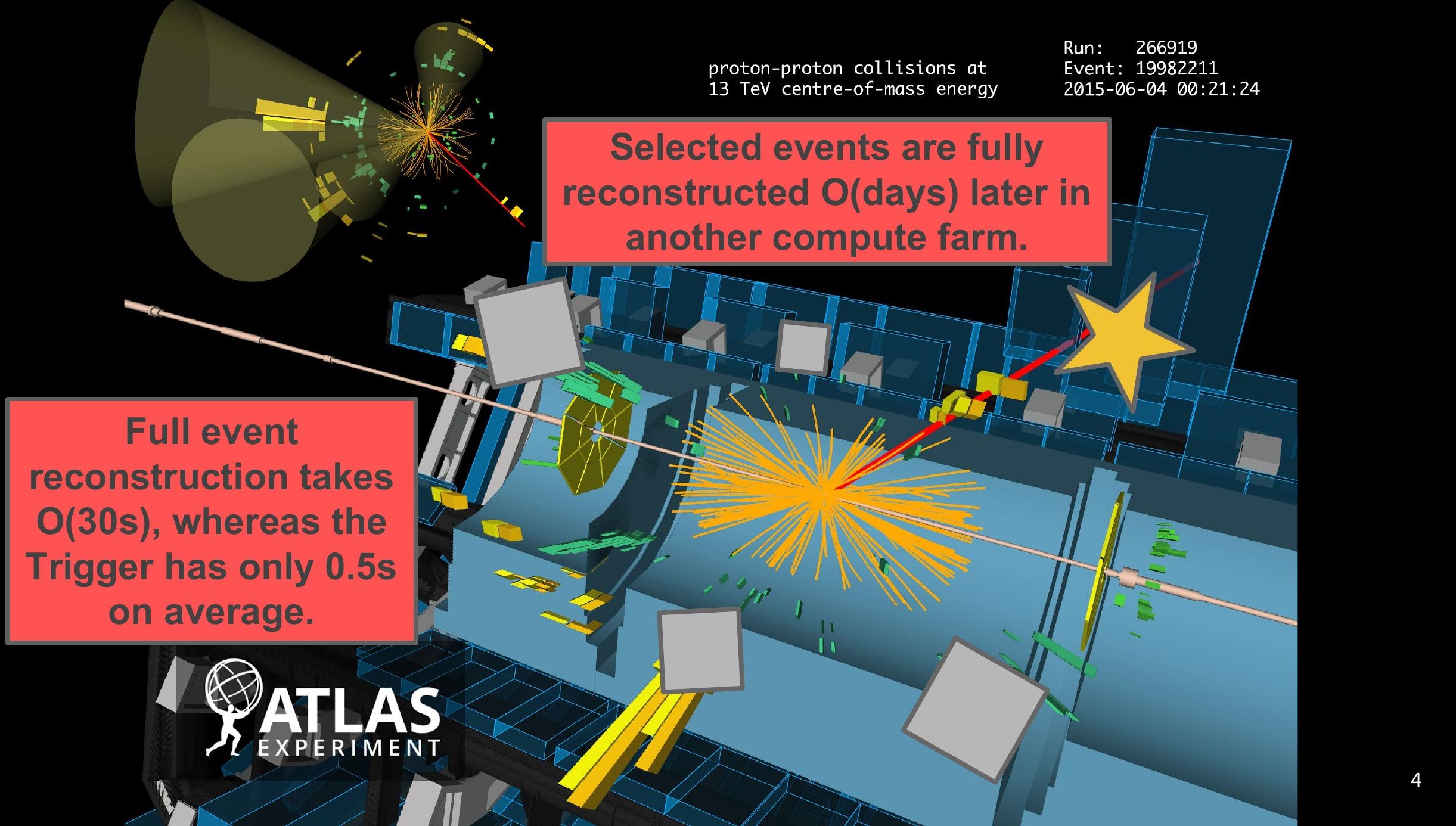




\section{Key Principles of the Trigger}

\section{Region of Interest}

- Regional Reconstruction

○ We cannot look at all 1.6 MB of every event due to bandwidth

○ Restrict to running reconstruction algorithms within

Regions of Interest, identified in the 1st level hardware trigger.

- Early Rejection

- Split reconstruction up into multiple Steps.

○ Filtering occurs after each Step via Hypothesis Algorithms

- Early steps are fast, but coarse.

- Later steps take more time, but are detailed.

- Stop reconstructing an object as soon as it fails a selection at the end of a Step.

○ Stop reconstructing the event when all objects are rejected.

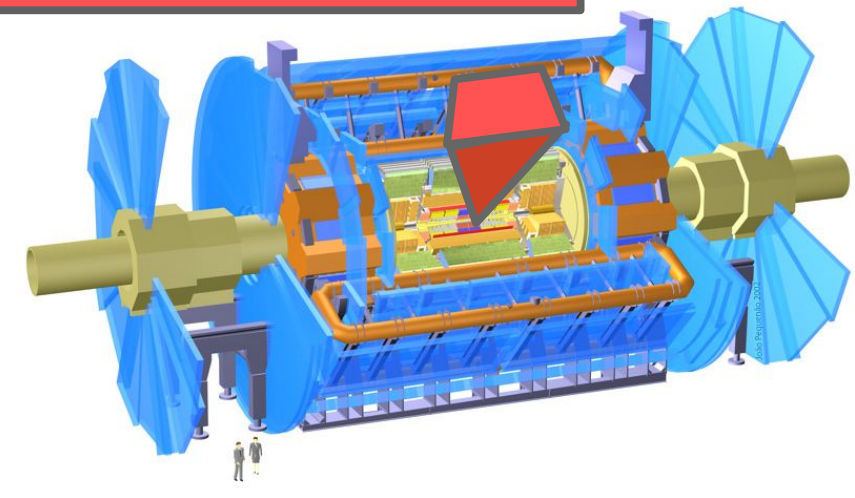

$$
\text { Stop reconstructing the event when all objects are rejected. }
$$

\section{Step 1}
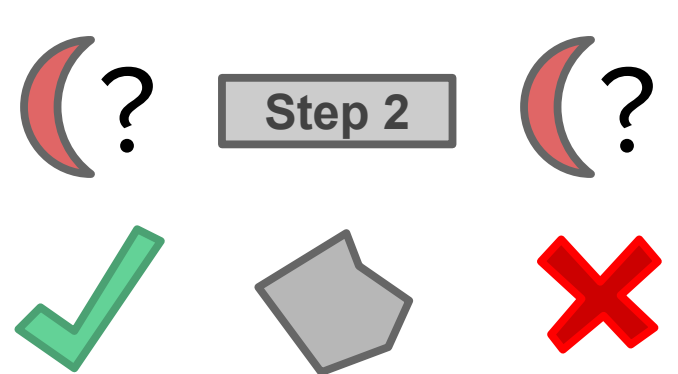

\section{Step 1}

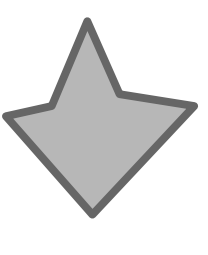

\section{Step 2}

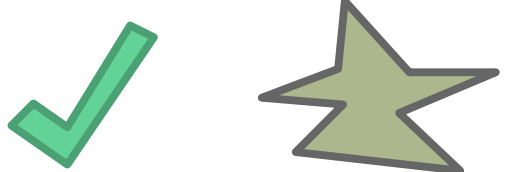

Hypothesis Algorithms

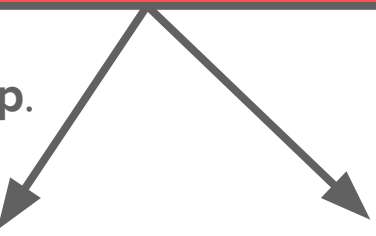

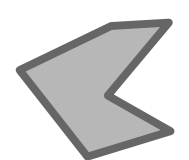
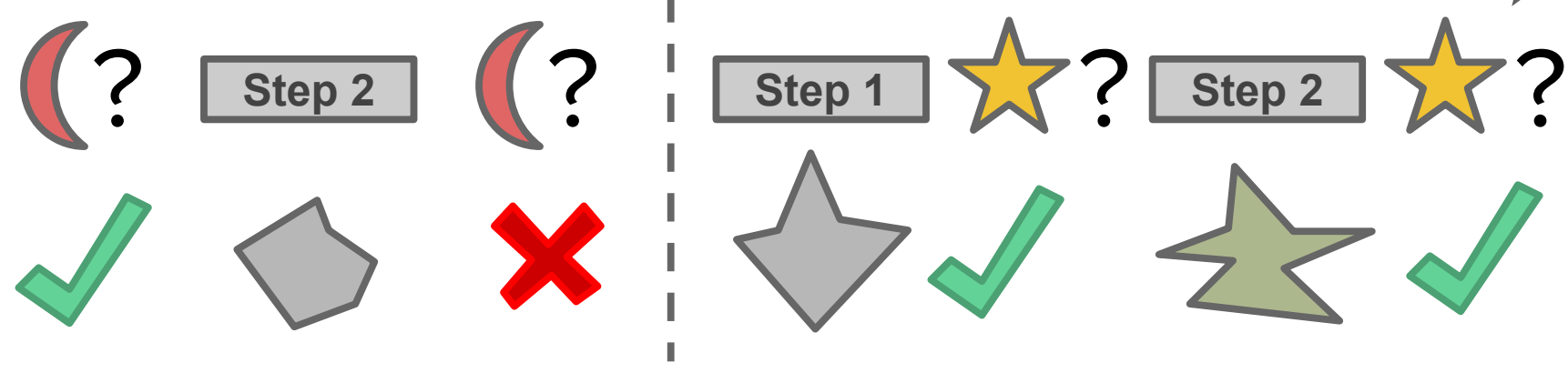

Step 3

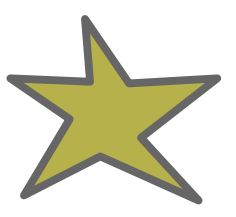

\&?

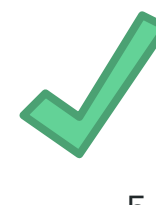




\section{Software Framework: Athena}

- The ATLAS Software framework, Athena, is built on top of the inter-experiment Gaudi framework (shared e.g. with the LHCb collaboration).

$\circ \quad \mathrm{C}++$ Algorithms, Services, Tools etc. with Python configuration.

- For each event a sequential list of algorithm executed. Algorithms are assumed to depend only on other algorithms scheduled earlier in the list.

- One common singleton Event Store handles transient and persistent data access.

- High Level Trigger uses custom

Top Sequence

steering, regular algorithms need wrapping.

- In the High Level Trigger, multi-core machines are currently utilised by forking the configured HLT process instance.

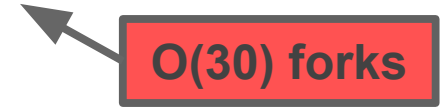

- Memory is shared with copy-on-write. Introduces overheads when pages are modified after the fork.
Event Store

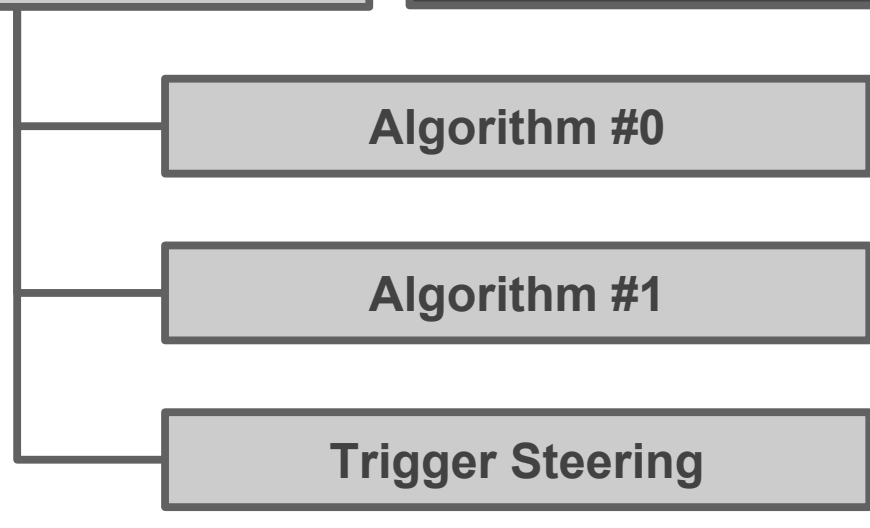




\section{Current Single Threaded Trigger Architecture}

- Object selections are encoded in Chains. Each step runs in serial.

\section{8: Over 1,300 active chains}
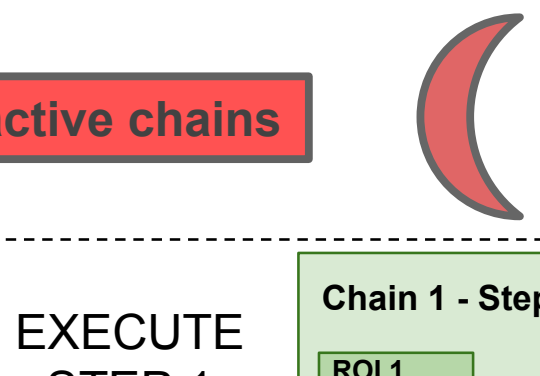
STEP 1

\begin{tabular}{|l|}
\hline Chain 1 - Step 1 \\
\hline Rol 1 \\
\hline Alg A \\
\hline Alg B \\
\hline Hypo C \\
\hline
\end{tabular}

Chain 2 - Step 1

Alg D

Alg $\mathrm{E}$ Hypo F
Cached result used 


\section{Current Single Threaded Trigger Architecture}

- Object selections are encoded in Chains. Each step runs in serial.
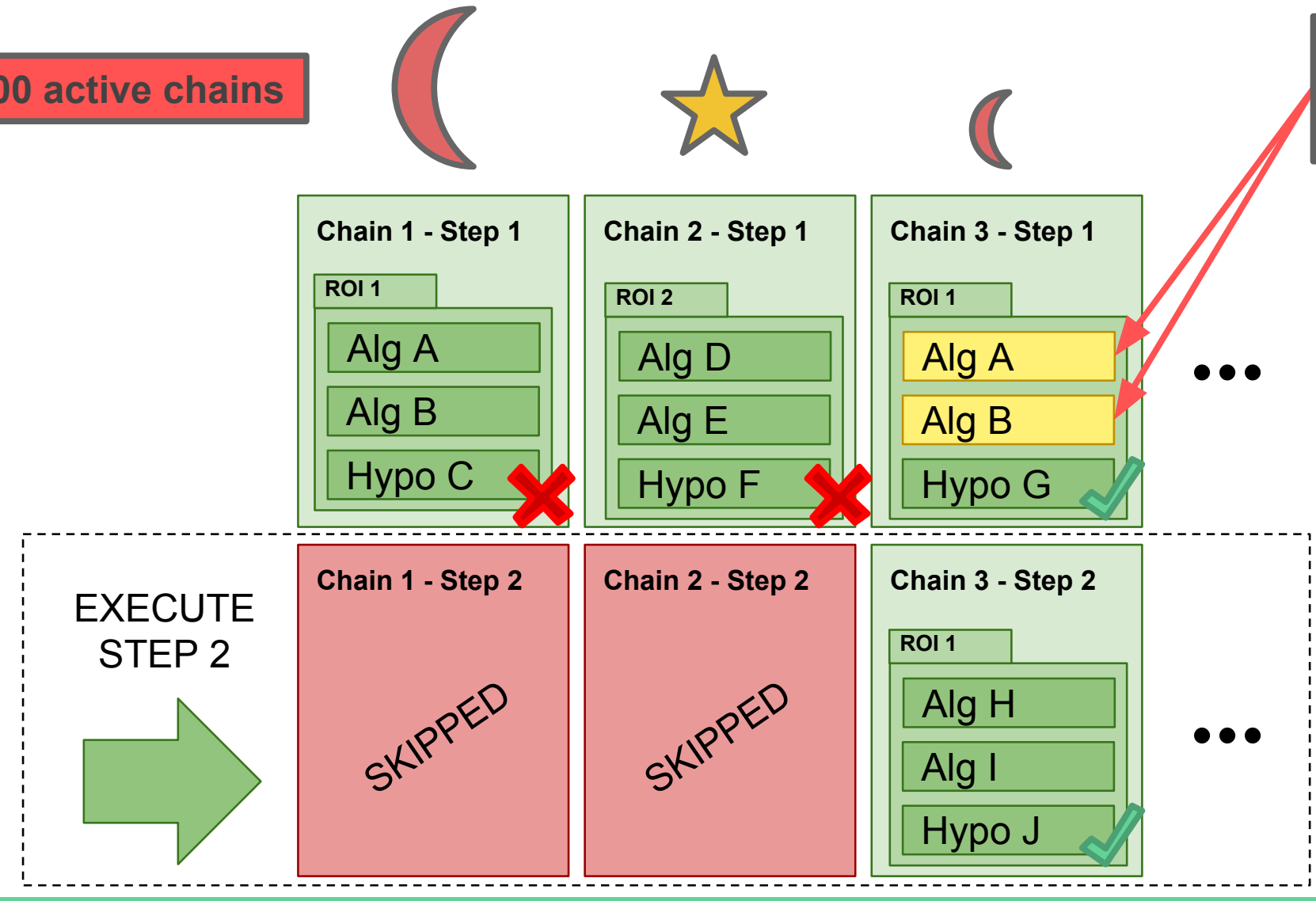

Cached result used

$\bullet$ 


\section{Moving on to Athena Multi-Threaded}




\section{Future Athena Multi Threaded (MT)}

- AthenaMT is built on the Gaudi Hive (Intel TBB) multi-threaded architecture.

- Offers Intra-Event parallelisation.

- The Algorithm Scheduler is configured with the Input- and Output-Data Handles of all algorithms. Builds a Data Dependency graph.

- Multiple algorithms within an event can run in parallel, provided that their input Data Handles (if any) are available.

- Offers Inter-Event parallelisation.

○ Multiple events may be being processed simultaneously: "in flight".

- Optimal memory efficiency if all algorithms are re-entrant, i.e. stateless and able to run on multiple concurrent events (alternate: cloneable).

- Offers In-Algorithm parallelisation.

- Algorithm authors may make use of e.g. parallel for-loops.

Goal: Maximise memory efficiency \& keep all threads busy. 


\section{AthenaMT}

\section{Event 3}

\section{Event 0}

Event 4

Thread 0

Thread 1

Thread 2

Thread 3

!

Event 1

Event 2 


\section{AthenaMT Data Dependencies}

- Suppose three types of selection:
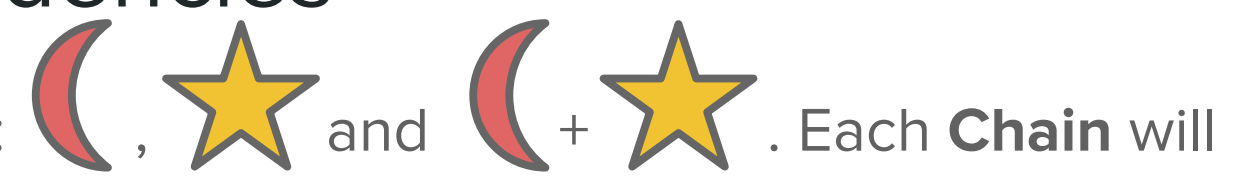
follow one of these three paths, with the chain's configuration controlling object quality \& object size requirements.

- We build a dependency graph of the algorithms required to perform the reconstruction. Like in the current system, it is split into different steps.

- Three classes of algorithm are used to control the execution.

○

Filter Algorithm

Always runs at the start of each step. Responsible for implementing Early Rejection. Returns a boolean Filter Decision to the Gaudi MT Scheduler.

$\circ$ Input Maker Algorithm Provides concrete starting point for reconstruction algorithms. Responsible for restricting reconstruction to Regions of Interest.

○ Hypothesis Algorithm Executes hypothesis testing for all active Chains. Provides input to next Step's Filter(s). 


\section{Data Dependencies}

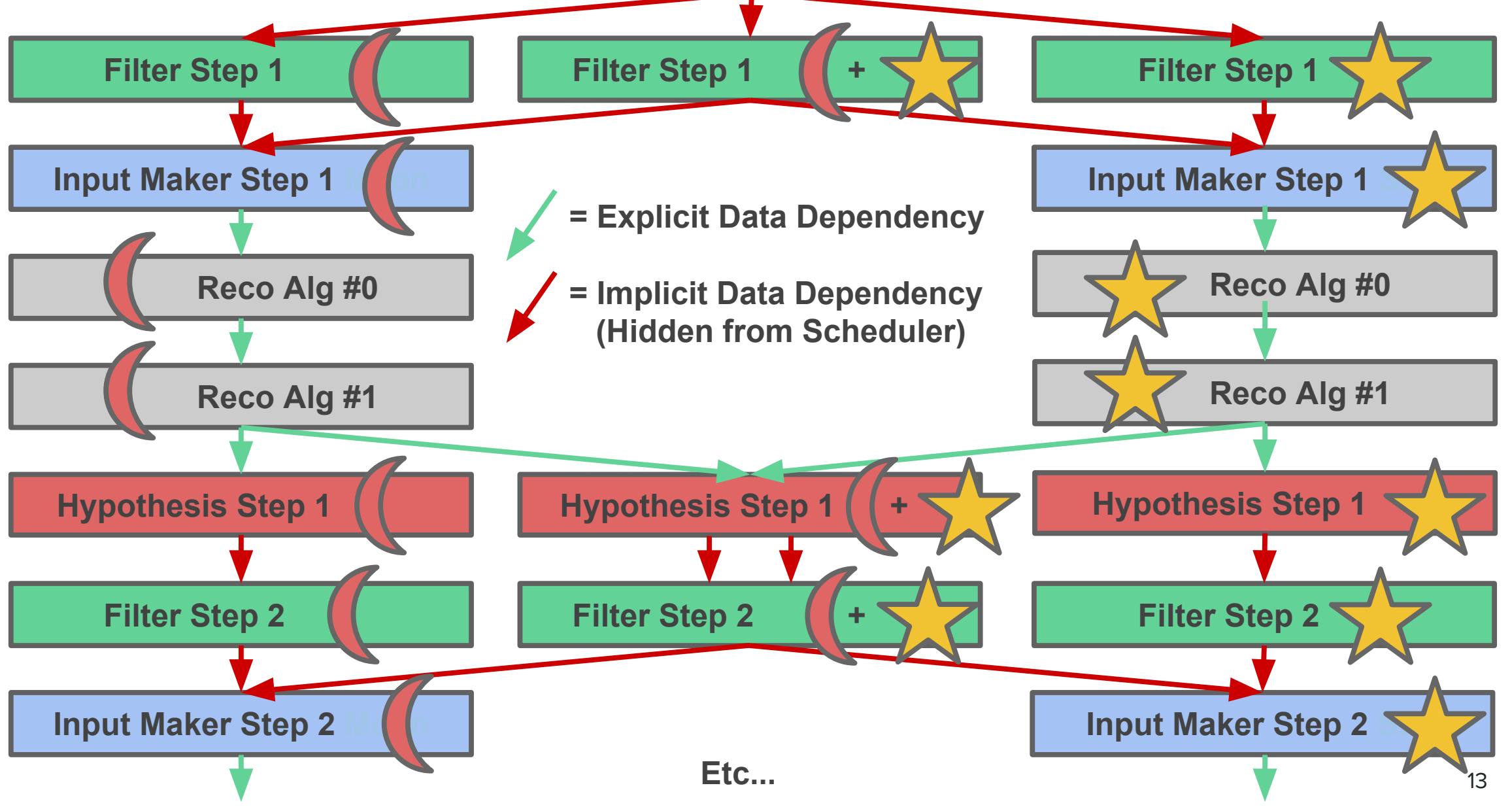




\section{Data Dependencies}

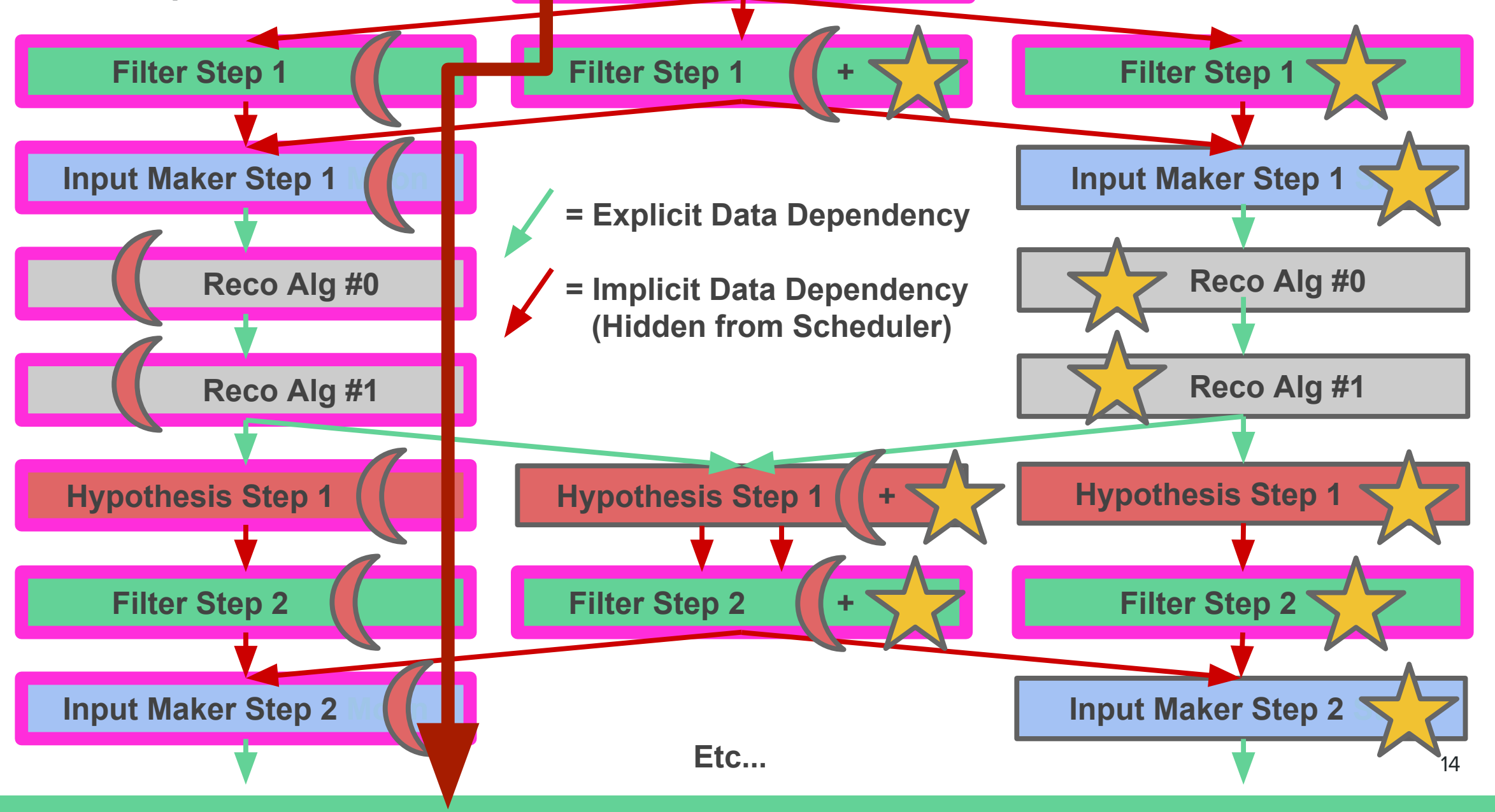




\section{Data Dependencies}

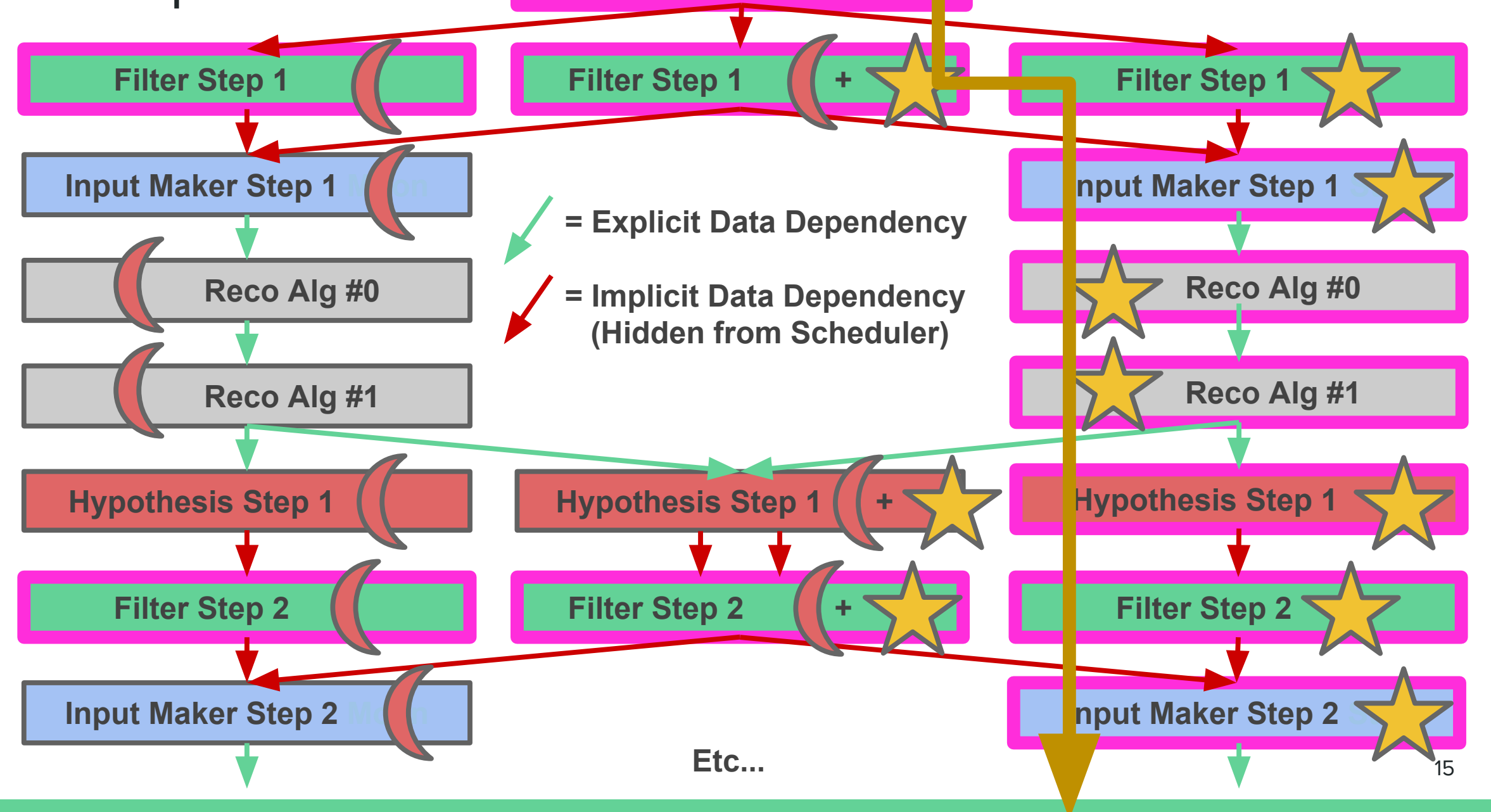




\section{Data Dependencies}

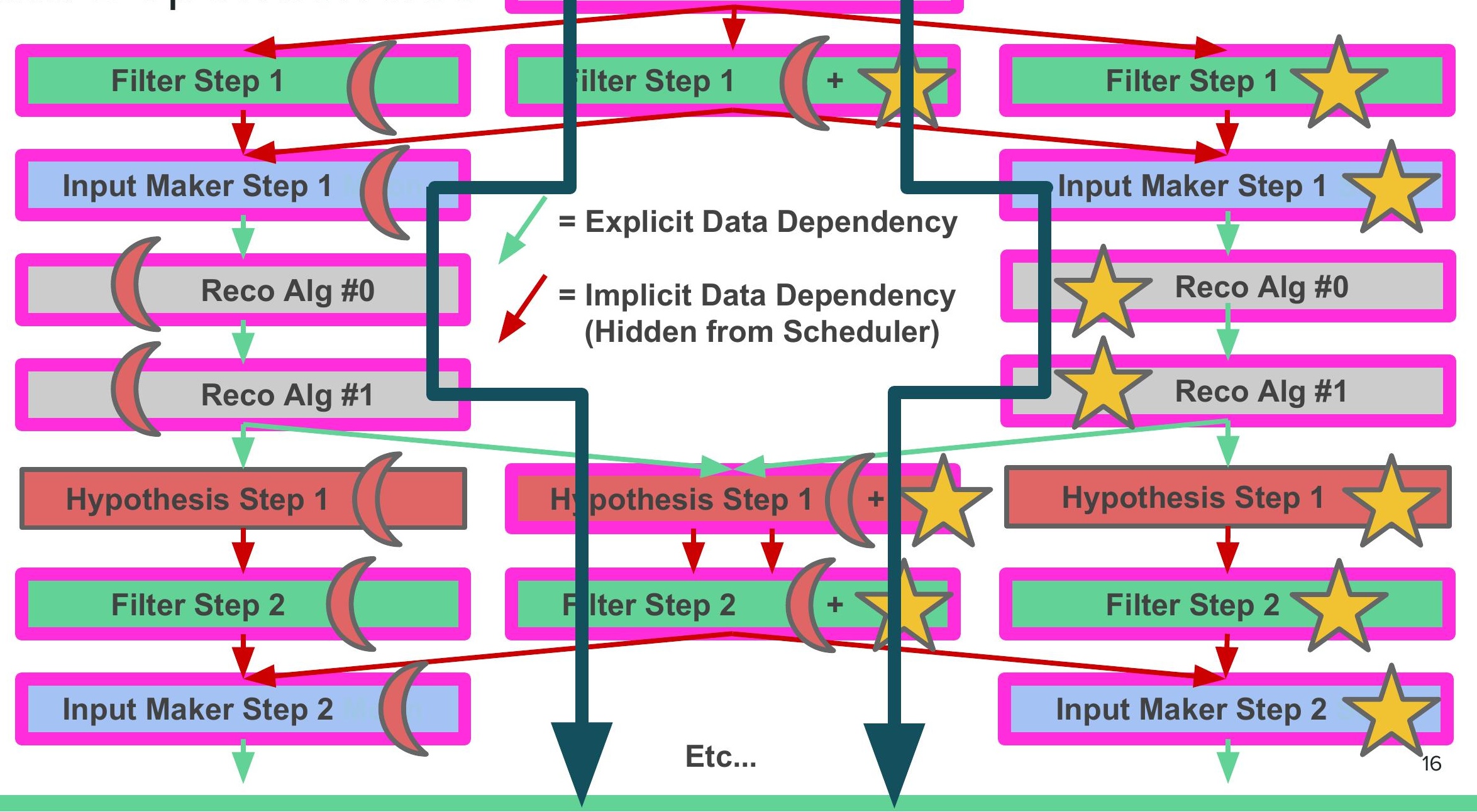




\section{Control Flow}

- The Data Dependency graph on the previous slide is not enough on its own.

- We need a mechanism to stop Filter Step 2 from running before Hypothesis Step 1 has executed and returned.

- Introduce a second Control Flow graph, built from two types of node (Sequencers).

- The OR node cannot exit early. It will schedule all of its children to execute in parallel, and return the logical OR of its children's filter decisions upon completion.

- The AND node can exit early. It will schedule its children to run AND sequentially, one after the other. Should a child return False, the sequencer will halt execution and return False to its parent. Otherwise, it will return the filter decision of its final child. 
Start

Etc...

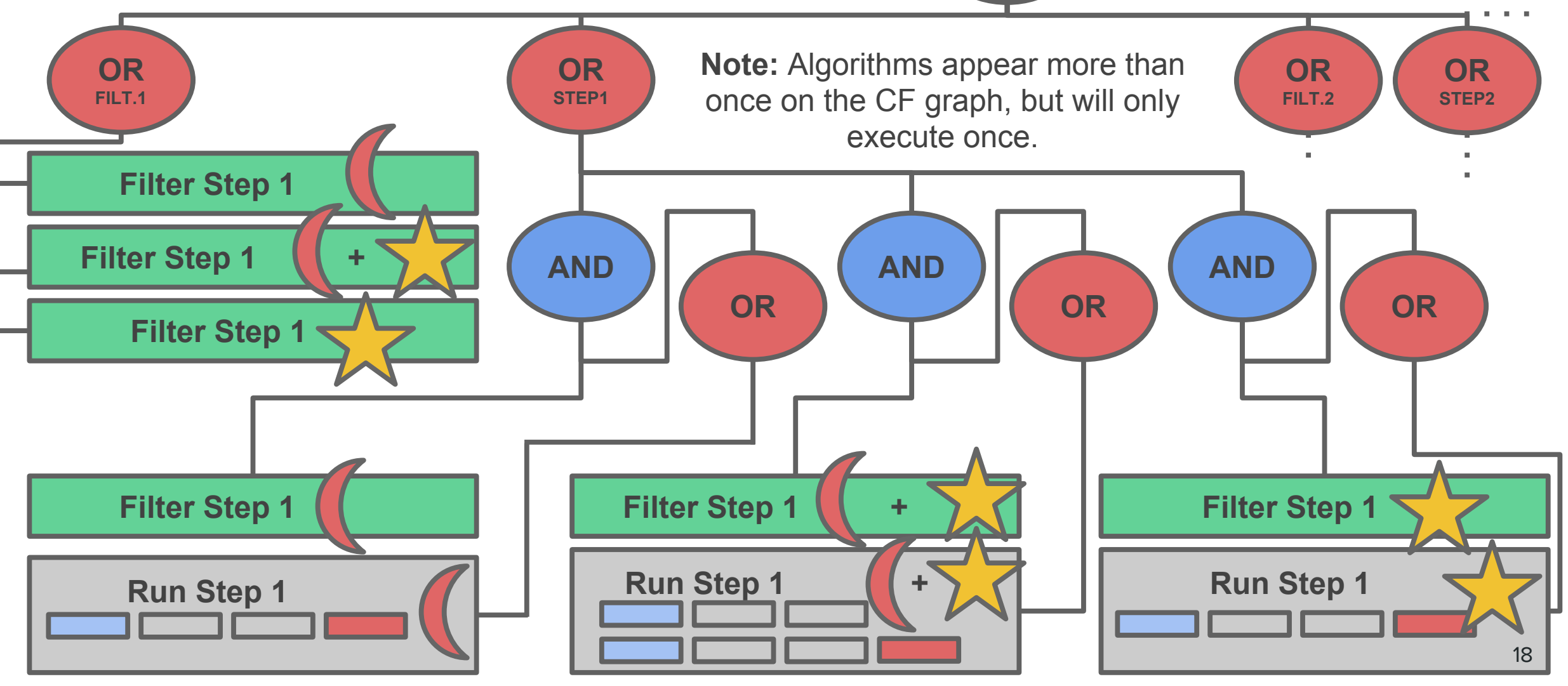


Control Flow
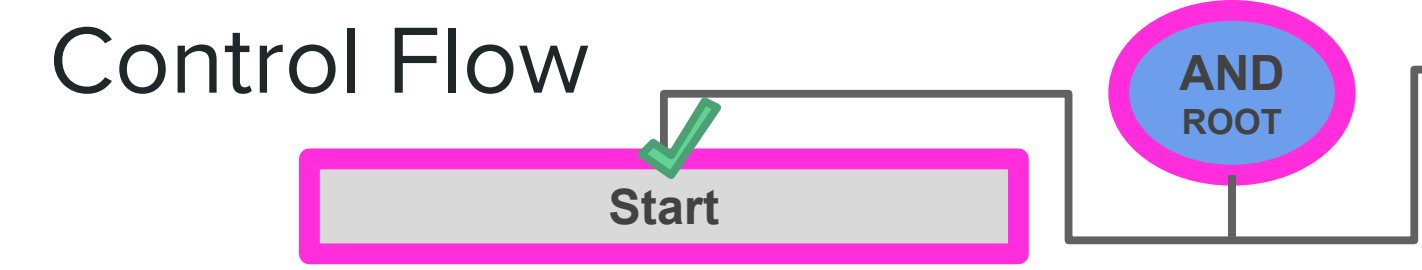

Note: AND Nodes execute left-to-right

Etc...

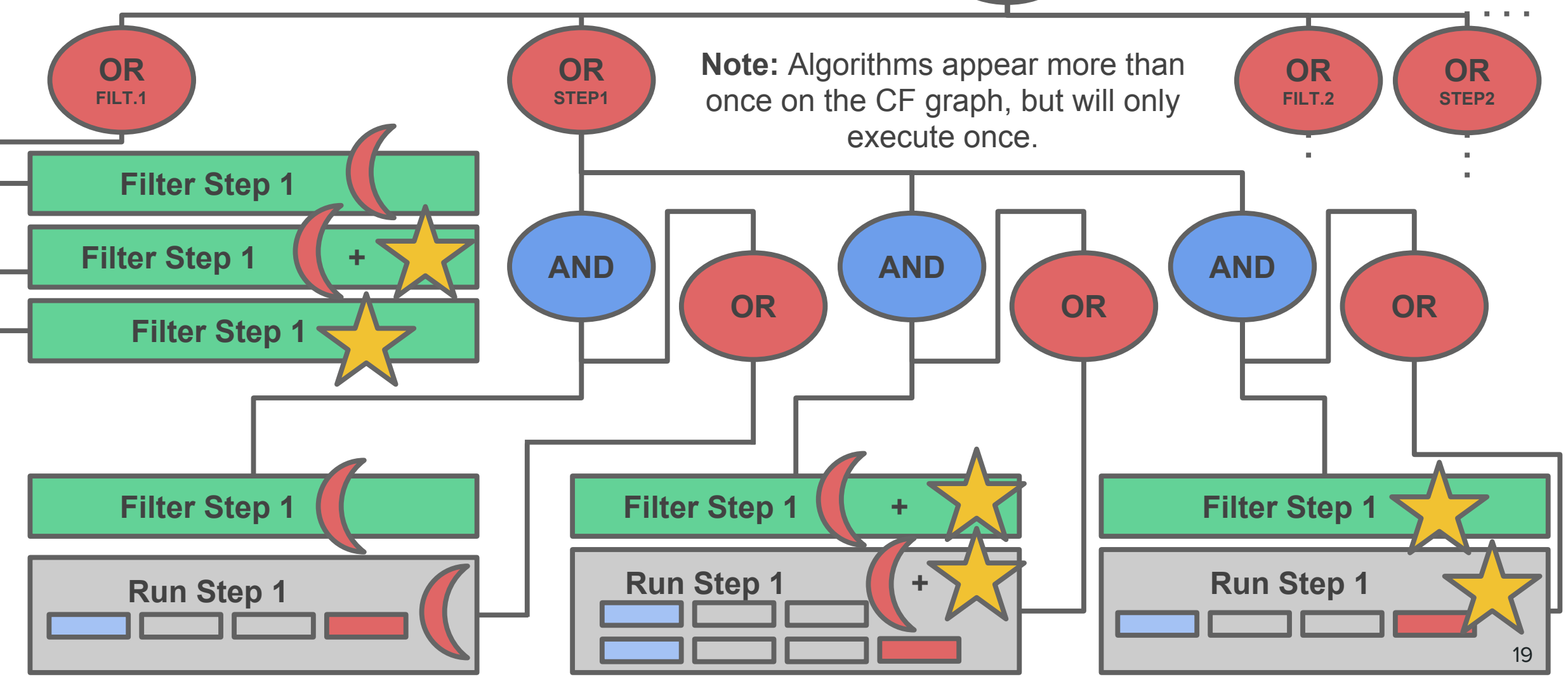




\section{Control Flow}

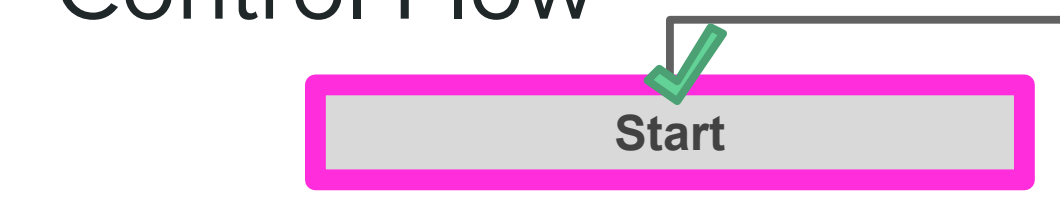

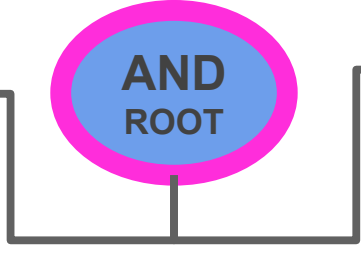

Note: AND Nodes execute left-to-right

AND STEPS

Etc...

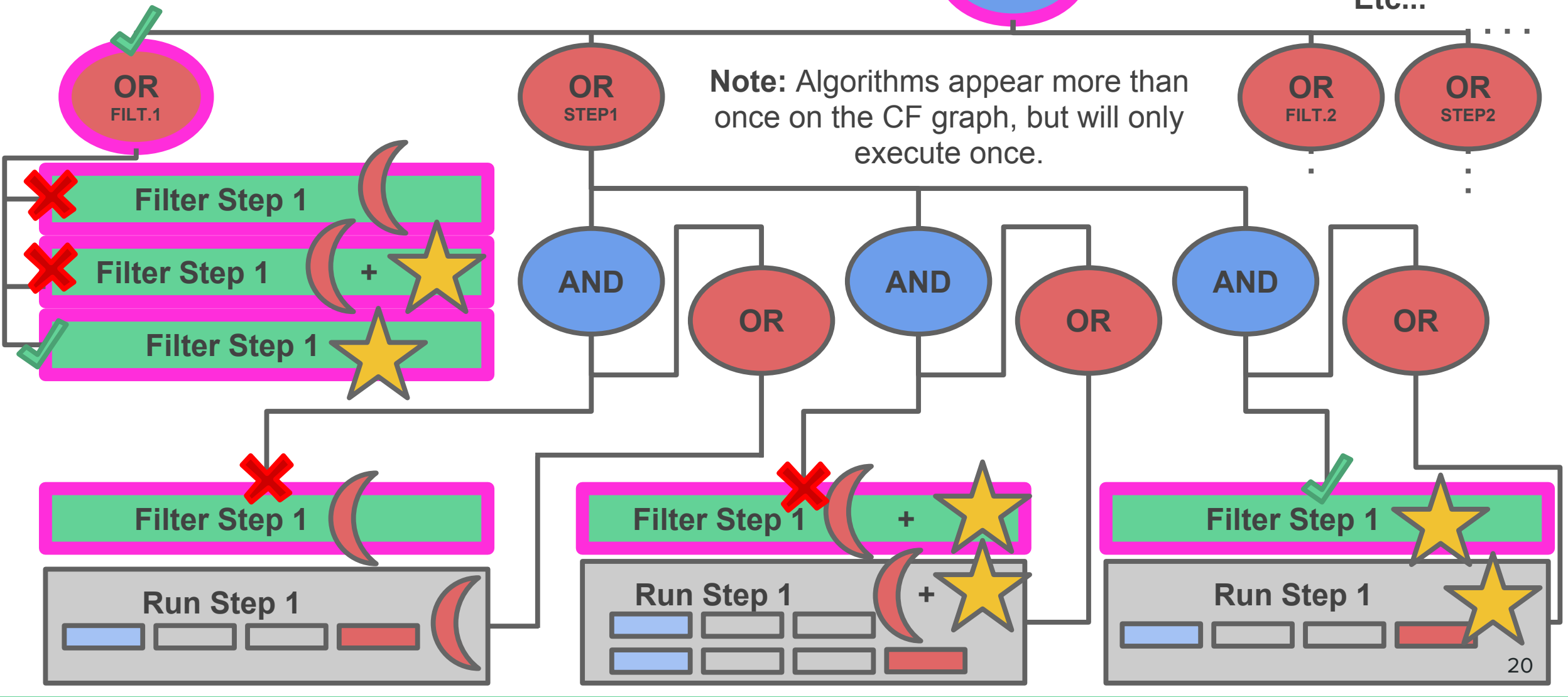




\section{Control Flow}

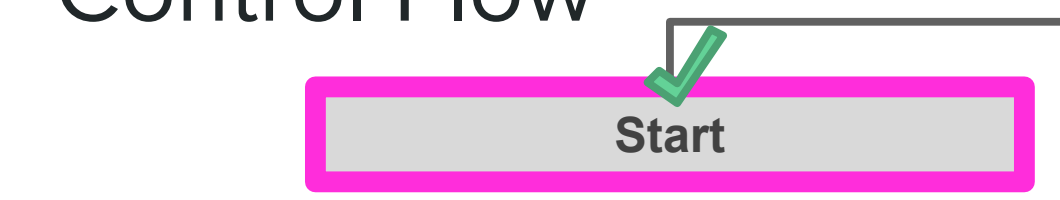

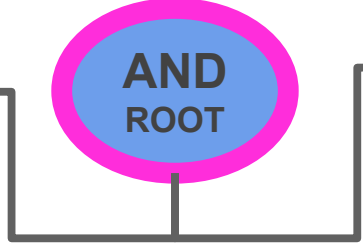

Note: AND Nodes execute left-to-right

Etc...

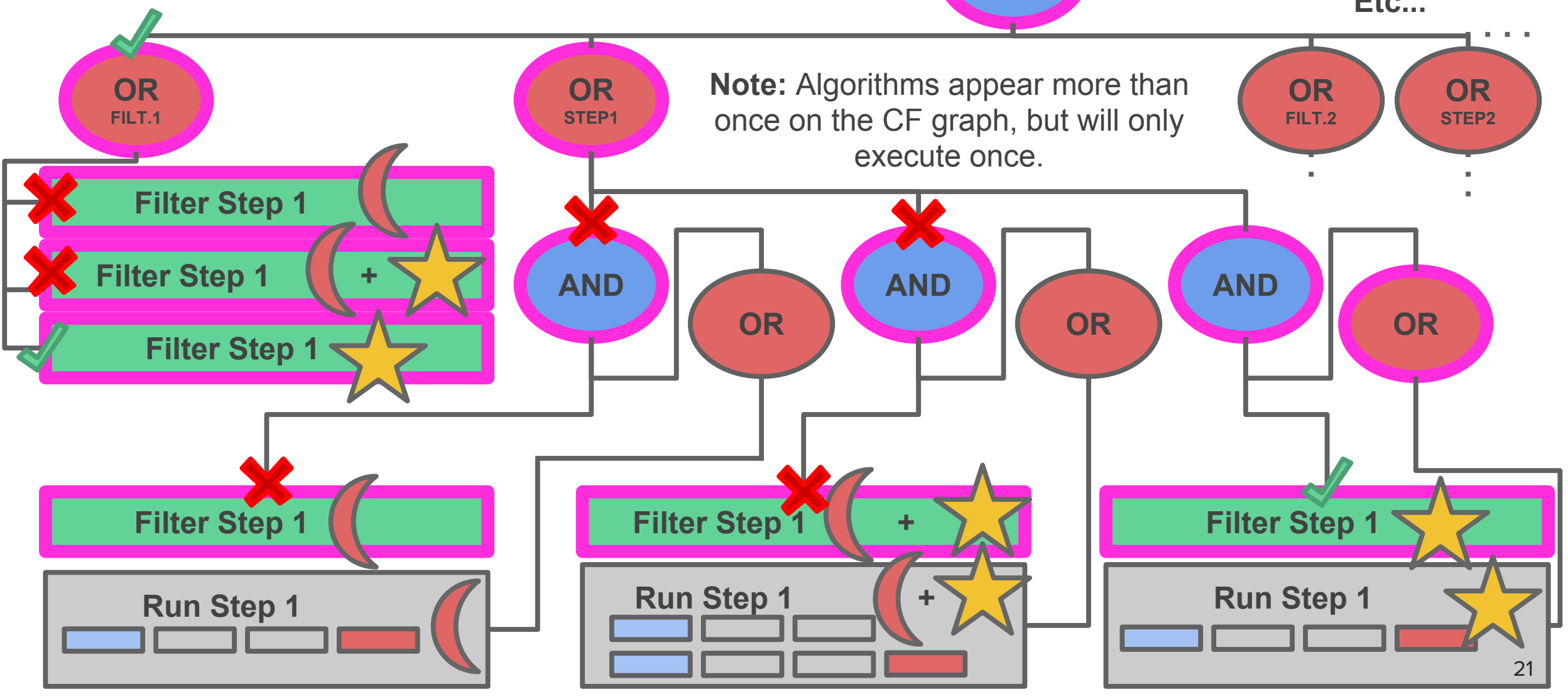




\section{Regional Reconstruction: Event Views}

- Gaudi Hive will allow each algorithm to execute at most once per event.

- But Regional Reconstruction requires algorithms to run once per Region of Interest.

- ATLAS Extension: Event Views.

- Spawn one Event View per Region of Interest, Schedule algorithms per View.

- Event View Implements the Event Store interface.

- Completely transparent to the algorithm. It does not know it's in a view.

- On completion, merge back to a single collection within the full event context.

Input Maker

Event Store

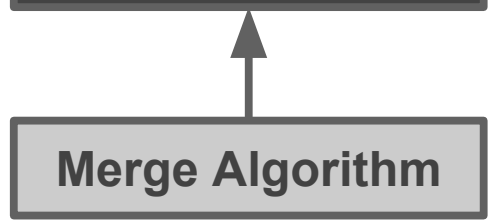

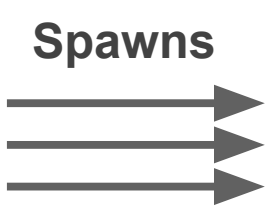

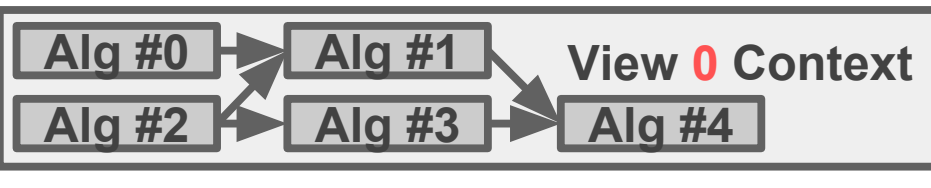

\begin{tabular}{|lll|l|}
\hline Alg \#0 & Alg \#1 & View 1 Context & Event Store \\
\hline Alg \#2 & & Alg \#3 & Alg \#4 \\
\hline
\end{tabular}

Merges
Event Store
Event Store

Event Store

\begin{tabular}{|lll}
\hline Alg \#0 & Alg \#1 & \multicolumn{1}{c}{ View 2 Context } \\
\hline Alg \#2 & Alg \#3 & Alg \#4 \\
\hline
\end{tabular}

Etc... 


\section{Wrapping Up}

- AthenaMT project will allow for greater scalability to the reality of a multi-core world where memory per core comes at a premium.

- The two key principles of trigger processing: Early Rejection and Regional Reconstruction are implementable in native Gaudi Hive using a combination of Data Dependencies, Control Flow and ATLAS' Event View extension.

- Will provide greater unification of the framework by removing Trigger-specific steering and wrappers.

- Working on implementation of framework and physics selections for use in LHC Run 3 in 2021. 


\section{Backup}




\section{Control Flow \& Data Dependencies - In Words}

- In this design, all Filter algorithms run first in a Step.

$\circ$ Check if any Chains which utilise the filter are still active and return True if so.

- If all Filters in a Step return False the parent OR node will also return False: implements Early Rejection.

- Reconstruction algs are unlocked by the Filters which still have active Chains.

- Algorithms can be unlocked by multiple Filters, they will still only run once.

- Input Maker algorithms have no explicit Input Data Dependencies, they will be scheduled to execute first when a Step is unlocked.

- Reconstruction Algorithms consume the explicit outputs of the Input Maker.

- The Hypothesis Algorithm is the terminal Data Dependency. It tests the Hypothesis of each active Chain against the reconstructed objects.

- Once all unlocked Hypothesis Algorithms return, the next Stage is unlocked.

- All Filter algorithms read in the previous stage's Hypothesis and checks if any Chains are still active. 